\title{
A rare case of metastatic lung cancer to the bladder
}

\author{
William Karle, $B S,{ }^{*}$ Yagil Barazani, $M{ }^{\dagger}{ }^{\dagger}$ Basir Tareen, $M D^{*+}$
}

*Albert Einstein College of Medicine, New York NY; 'Beth Israel Medical Center, New York, NY

Cite as: Can Urol Assoc J 2012;6(4):E147-E149. htrp://dx.doi.org/10.5489/cuaj.11129

\section{Abstract}

Cancer metastasis to the bladder from non-contiguous sites is very rare. Lung metastasis to the bladder is even more uncommon, with an extensive literature search identifying only four documented cases in the past 20 years. Of these four cases, only one was from lung adenocarcinoma. In this report, we present the case of a 66-year-old male with known lung adenocarcinoma found to have the incidental finding of a bladder mass upon computed tomography imaging. Histochemical staining of samples from the bladder confirmed metastatic dissemination from the primary lung malignancy.

\section{Introduction}

Cancer metastasis to the bladder from non-contiguous sites is very rare. Studies have shown that between $2 \%$ and $12 \%$ of all bladder tumours are secondary neoplasms. ${ }^{1,2}$ Lung metastasis to the bladder is even more uncommon, with an extensive literature search identifying only four documented cases in the past twenty years. ${ }^{3-5}$ Secondary neoplasms in the bladder have about the same incidence as non-transitional cell carcinoma (TCC) - both with an incidence ranging from $2 \%$ to $17 \% .^{1,2,6-8}$ However, when secondary neoplasms are found within the bladder, the primary lesion is almost always from a contiguous site. ${ }^{7}$ Occasionally, distant spread can occur from primary malignancies originating in the stomach, skin and, least likely, the lung. Unfortunately, gross examination is rarely able to differentiate between these two types of bladder cancer. ${ }^{8}$ Due to the usually low level of clinical suspicion and to the difficulty in histologically differentiating primary non-TCC of the bladder from secondary neoplasms, making an accurate diagnosis is often difficult. ${ }^{9}$ Therefore, very few studies of metastatic spread from primary lung cancer to the bladder have been described.

\section{Case report}

A 66-year-old male with progressive stage 4 lung adenocarcinoma was incidentally found to have severe hydronephrosis of the left renal collecting system, as well as a markedly thickened bladder wall measuring up to $3 \mathrm{~cm}$ at the left ureterovesical junction (UVJ) (Fig. 1). At the time of presentation, the patient denied gross hematuria, dysuria, flank pain or any other genitourinary symptom.

Physical exam was unremarkable. Urinalysis revealed 3 red blood cells per high power field and urine cytology was negative for malignancy.

Office cytoscopy showed a solid mass along the left trigone. The patient later underwent cystoscopy with biopsy under anesthesia, which again demonstrated diffuse erythematous and bullous changes of the bladder mucosa, particularly at the left trigone area consistent with the computed tomography scan. The right ureteral orifice was immediately visualized, however the left ureteral orifice was entirely obscured. Deep biopsies were obtained from the left trigone, posterior bladder wall and right lateral bladder wall.

Histological examination of left trigonal biopsies identified an invasive poorly differentiated carcinoma with abundant mucin located sub-epithelially and infiltrating into the muscularis propria. Interestingly, the adjacent muscularis propria and bladder mucosa appeared unremarkable (Fig. 2). The poorly differentiated nature of this neoplasm, combined with the overlying benign urothelium, was suggestive of a non-urothelial origin. Immunohistochemical staining of tumour cells was strongly positive for TTF- 1 and CK7, while high molecular weight cytokeratin was focally positive. P63, CK20 and androgen receptors were negative. These findings were consistent with metastatic lung carcinoma of the bladder. From this data, the final diagnosis was made of metastatic lung adenocarcinoma to the bladder.

Given his widespread pulmonary and bladder metastases, the patient opted to undergo palliative chemotherapy with gemcitabine and carboplatin. Despite this regimen, the 


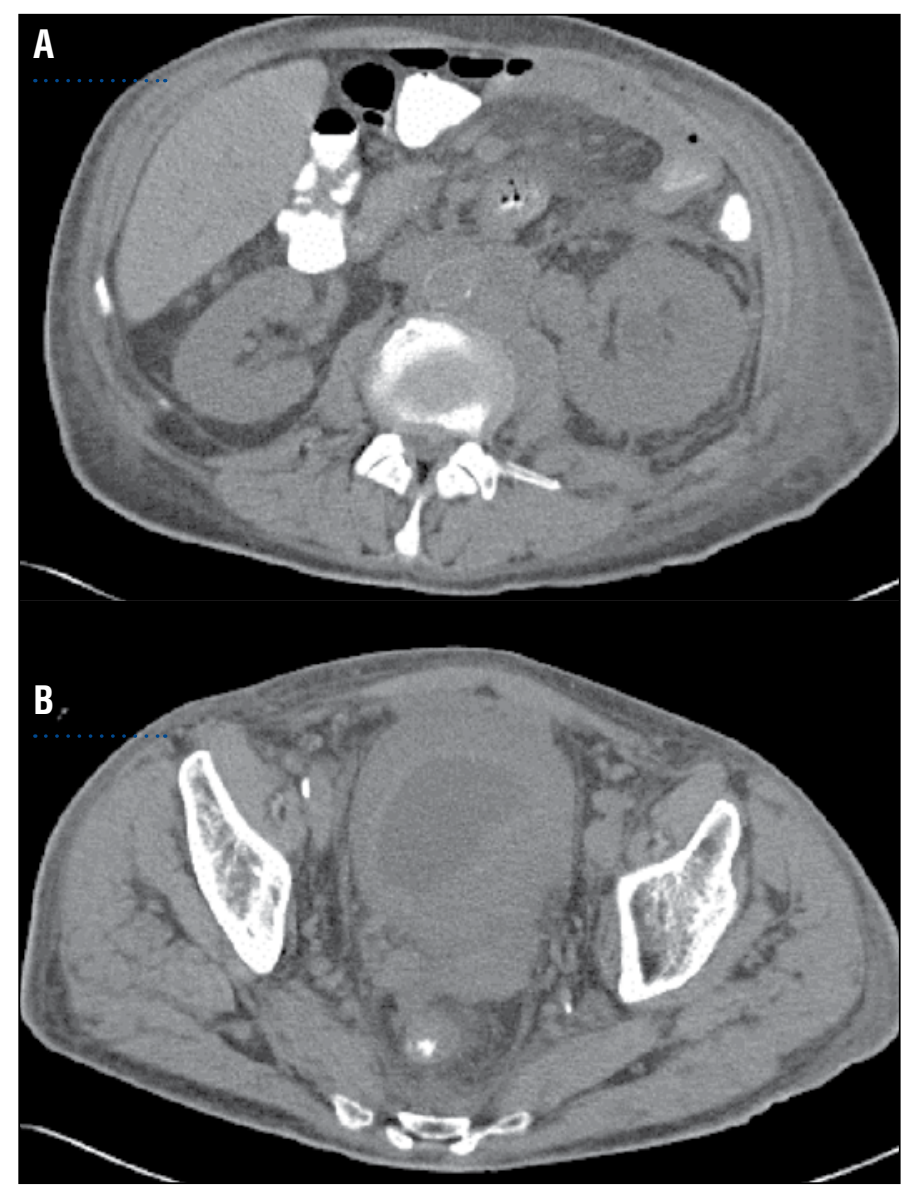

Fig. 1. Noncontrast computed tomography scan of the abdomen/pelvis demonstrating (a) left hydroureteronephrosis leading to the level of the ureterovesical junction (UVJ) and (b) a urinary bladder that is markedly thickwalled and measures approximately $3 \mathrm{~cm}$ in thickness at the region of the UVJ.

patient exhibited progression of pulmonary metastases on serial chest radiographic imaging at 2- and 4-month intervals. Given his deconditioned state and the extent of his metastases, the patient at this point enrolled in a hospice program. At this time, he is alive 5 months post-transurethral resection of bladder tumour, however is not currently undergoing any further treatment.

\section{Discussion}

In patients with secondary bladder cancer, hematuria and pelvic pain are often the presenting symptoms, if they are not found incidentally. ${ }^{10}$ One of the most recent case reports of lung adenocarcinoma metastasizing to the bladder was in 2006 with a patient who initially presented with pain and bilateral hydronephrosis. This patient died 8 months after the diagnosis, despite chemotherapy. ${ }^{4}$ In our patient's case, metastatic spread of lung cancer to the bladder was discovered prior to the onset of clinical symptoms. This is common, as infiltration of the mucosa or ulceration is required

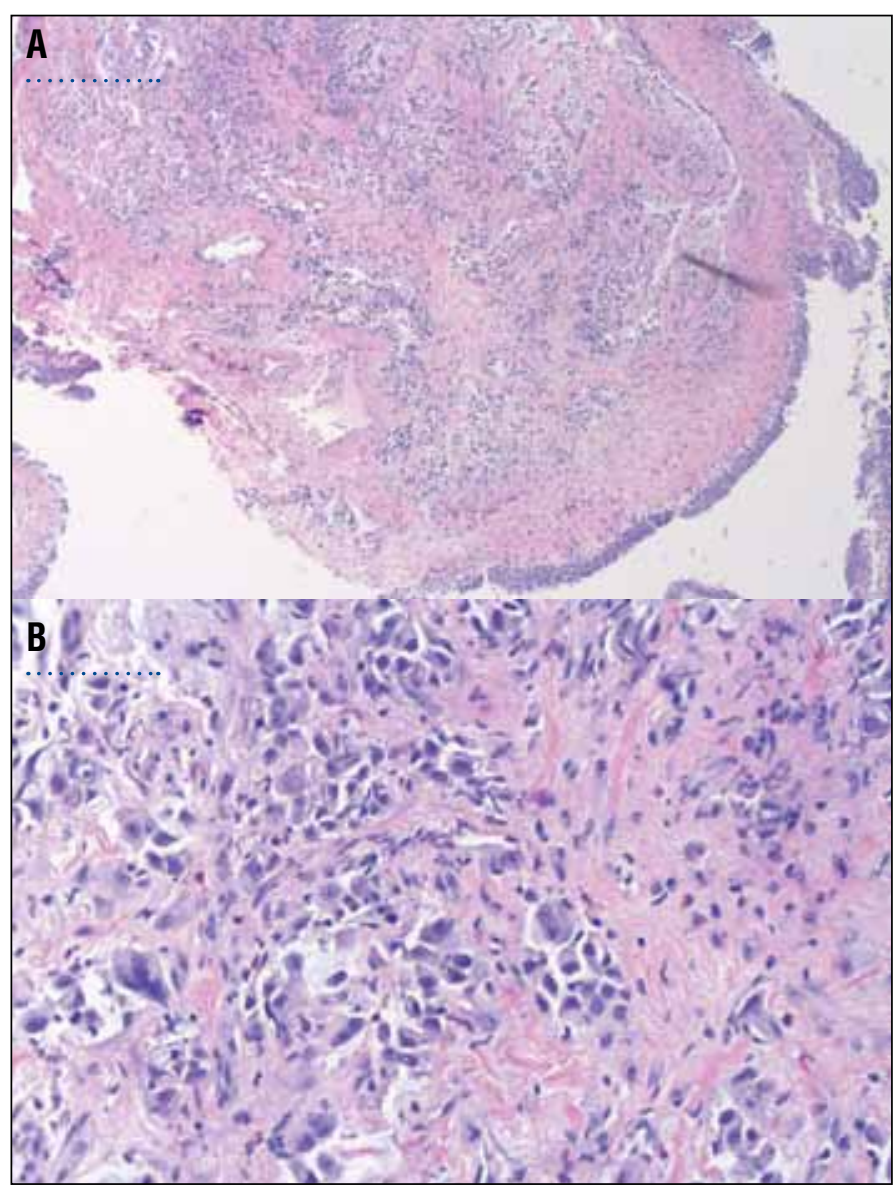

Fig. 2. Hematoxylin-eosin stain of a bladder biopsy showing (a) normal urothelium bottom right with tumor infiltrating stroma at the centre and (b) high power field of poorly differentiated carcinoma demonstrating infiltrating cells that are pleomorphic with high nuclear/cytoplasmic ratios.

before hematuria becomes appreciable. ${ }^{11}$ A high index of suspicion of lung metastasis to the bladder is often difficult to determine on histological examination and histochemical staining. This was further supported by the presence of stage 4 , widely metastatic lung cancer on preoperative imaging.

In all cases of non-TCC of the bladder, histochemical staining should be performed given the potential for a secondary neoplasm. This is much easier to accomplish if a primary cancer has already been discovered and specific markers can be used. Examples of specific histochemical analysis include placental S100, GATA3 and napsin A, which were used in a previous case of lung adenocarcinoma metastasizing to the bladder. ${ }^{5}$ With high suspicion, primary sites of cancer in the colon, prostate, rectum and cervix should be sought, as they are the most likely culprits of direct spread. If not already standardized at the providing institution, all patients with non-transitional cell tumours in the bladder should receive imaging studies of the chest, abdomen, pelvis, and bones not just for staging, but also to rule out the presence of a possible primary cancer located 
elsewhere. Diagnosing primary versus secondary neoplasms of the bladder is extremely important, as primary cancer is often curable with cystectomy and/or chemotherapy, while secondary neoplasms are typically treated only with palliative chemotherapy. We believe that due to the difficulty of histologically diagnosing secondary bladder cancer from primary non-TCC, cases of secondary bladder neoplasms may go undiagnosed and the true incidence of this entity may therefore be under-reported.

\section{Conclusion}

We presented a rare case of metastatic adenocarcinoma to the bladder. While, secondary malignancies of the bladder are difficult to distinguish from non-transitional cell primary bladder cancer, both have nearly the same incidence. When confronted with a non-transitional cell cancer of the bladder, a combination of clinical suspicion, immunohistochemistry and routine histochemical examination should be used to differentiate its origin. An even higher level of suspicion and more thorough investigation are warranted if a known primary cancer already exists in the patient.

Competing interests: None declared.

This paper has been peer-reviewed.

\section{References}

1. Melicow MM. Tumours of the urinary bladder: a clinicopathological analysis of over 2500 specimens and biopsies. J Urol 1955;74:498-521.

2. Bates AW, Baithun SI. Secondary tumours of the bladder. J Pathol 1998;184(Suppl): 40A.

3. García Freire C, Rodríguez-Rivera García J, Picallo Sánchez J, et al. Bladder metastases of pulmonary carcinoma. A report of 2 cases. Actas Urol Esp 1994;18:601-3.

4. Lan SK, Lin YS, Tzai TS, et al. A Rare Instance of Lung of Lung Cancer Metastasizing to the Urinary Bladder. JTUA 2006;17:63-6.

5. Raspollini MR, Comin CE, Crisci A, et al. The use of placental S100 (S100P), GATA3 and napsin A in the differential diagnosis of primary adenocarcinoma of the bladder and bladder metastasis from adenocarcinoma of the lung. Pathologica 2010;102:33-5.

6. Bates AW, Baithun SI. The significance of secondary neoplasms of the urinary and male genital tract. Virchows Arch 2002;440:640-7. http://dx.doi.org/10.1007/s00428-001-0549-x

7. Bates AW, Baithun SI. Secondary neoplasms of the bladder are histological mimics of nontransitional cell primary tumours: Clinicopathological and histological features of 282 cases. Histopathology 2000;36:3240. http://dx.doi.org/10.1046/i.1365-2559.2000.00797.x

8. Ito T, Wada T, Furusato M, et al. Prostatic involvement of bladder carcinoma. Nippon Hinyokika Gakkai Zasshi 1997;88:677-83.

9. Torenbeek R, Lagendijk JH, Van Diest PJ, et al. Value of a panel of antibodies to identify the primary origin of adenocarcinomas. Histopathology 1998;32:20-7.

10. Acino SM, Hampel N. Renal cell carcinoma presenting with gross haematuria from a solitary bladder metastasis. Eur Urol 1988;15:294-6.

11. Poulakis V, Witzsch U, de Vries R, et al. Metastatic breast carinoma to the bladder: 5-year followup. J Urol 2001;165:905. http://dx.doi.org/10.1016/S0022-5347(05)66562-7

Correspondence: Dr. Basir Tareen, Physician-in-charge, Urologic Oncology, Beth Israel Medical Center, Assistant Professor, Albert Einstein College of Medicine, Phillips Ambulatory Care Center, Suite 3A, 10 Union Square East, New York, NY 10003; BTareen@chpnet.org 